\title{
Multi-Disciplinary Scientific Approach to Detection of Mass Repeated Fatal Errors in Standard Medical Orthopedic Processes
}

\author{
Antonín Cuc* \\ The International Expert in Occupational Health and Safety, Czech Republic \\ *Corresponding author: Antonín Cuc, The International Expert in Occupational Health and Safety, Na Míćánkách 435/1,101 00 Prague 10, Czech \\ Republic
}

Submission: 些 February 16, 2018; Published: 些August 21, 2018

\section{Editorial}

The frequency of fatal medical errors in Diagnosis and choosing the right individual treatment is tragic fatal by $10 \%$ of all workflow-today's ex-waiting fatal medical mistakes in OECD by The World Research Message WHO 2010. This means an immense amount of unnecessarily severely injured patients annually, with a substantial reduction in the rest of their lives for many years in all OECD countries. I very much appreciate the findings of medicine in the US, where it has been proven in 2016 that medical errors are the third most common cause of enforced Patient death in the US, although it is very difficult to demonstrate from standard structural patient medical records. I have therefore decided, on the basis of the standard criminalistic multidisciplinary methods used in the United States to analyse catastrophic falls of each transport aircraft, it was my origin inspiration: - to demonstrate how similar criminal analyses of statistical Patient samples can be performed a key to description random clusters of patients with comparable orthopedic diagnosis with comparable standard choice treatment with a comparable choice of orthopedic implants with detection many false similar repeated fatal medical mistakes.

The basis of the demonstrative approach is the problem of a model description of the history of at least One Statistical Unit - the elementary first case of at least one patient in the selected "first learning sample". We are begin with FIRST more explanatory case description of the Orthopedic case in next Clusters - with this new patented approach, Utility model S_T_A_R_S-Statistical Retrieval and Search of sufficient information to Mass reuse for similar repeated strategic decision making, it opens a crucial World discussion among all the members of the evaluating multidisciplinary scientific next working team-i.e., criminologists, orthopedists, radiologists, forensic doctors, patients, lawyers, statistics, biostatistics, experts in databases for the Clusters from clever selected records from used EHRs.

Experienced and well-structured motivated qualificator decision team by patented principles Utility model "S_T_A_R_S“, $21532 \mathrm{CZ}, 2010$. The problem of proper collective decision making in a team that uses multidisciplinary scientific approaches should be based on the reasonable professional and well-motivated staffing of the entire team, but they must go through a common initial "run-in" to make clear what decisive phenomena they must be able to recognize to evaluate and how to learn from the individual evaluations of the individual team members to come to the joint partial conclusions and to the final interpreting statistic representative conclusions, estimates of statistic parameters with statistic accuracy and statistic defined reliabilities - on the individual monitored phenomena, on the critical illegal situations of the patient Health care, the individual statistical units evaluated ... i.e. when checking the correct processing in diagnosis, checking the correct processing in choice the right individual treatment by the modern medical knowledge's interdisciplinary of Encyclopaedic Literature, assessing the illegal consequences of medical errors in diagnosis and in the choice of false individual treatment, evaluating the consequences of fatal unlawful medical errors by the decisions making on the Providers Health care and medical staff by the Health care for patient, the probable development of his / her status of Health, the growth of fatal patient risks in next time and a biostatic probable enumerable effects on the likely median estimate of shortening the rest of the patient's life for a significant number of years to legal financial satisfactions including the Life assurance for worse mental and Physical Health status, Loss of Patient Lives.

We should to understand to the casual history of a few individual Patient tragic growing the fatum to begin with next clever usage the Statistic method to detect similar medical mistakes EX POST, later so as for prevency to repeat medical mistakes EX ANTE. In Orthopady CZ, you never could take Members in Decision Research Team CZ , because all Orthopaedic Patients CZ there are living OR there are heavy injuring OR there are dying only unconditionally „LEGE ARTIS CZ", by the constant false meaning of all Providers Health care CZ and medical staff CZ, by the constant false meaning all Knowing Medical Institutes CZ, by the constant false meaning of Criminal Departments of Police CZ, by the constant false meaning of the all Justice CZ, by the meaning of the Heads of all Penalty Offices 
CZ-The Patient could be a silent Victim CZ only, without the Human Rights for Life and juiced truth verified Criminal evidences by the justified Court trials.

We are living live in the legal State of Czech Republic, where all unnecessarily mutilated or dying orthopedic patients of CZ have no real civil human rights to use forensic technical evidences by Court trials CZ-that they have been silent needless Victims CZ of The Mass repeated illegal fatal medical procedures in orthopadic Diagnostics or Treatments-for example contrary Technician Requirements of implanting Laws EU/CZ for guaranty the legal usage Medical Implants with „CE“ marking by the Technician Requirements of the Directive 93/42/EEC Medical Devices in common Market EU/CZ since April 1, 2004.

Since there are never seen any fatal official medical errors in Czech Orthopaedics CZ, it is not real possible or desirable to detect or efficiency to prevent such massive medical orthopaedic errors in medical orthopadic workflow, namely we don't solve tragic Fatums of Patient CZ in the Czech Republic, we don't count the yearly needless Patient Victims CZ. We are taking the false definition, LEGE ARTIS CZ "regardless It has covered namely in Mass frequent repeated trivial technical medical fatal errors in Mass Medical processing CZ.

The needless Loss of Orthopedic Patient Life CZ has NONE of financial evaluating. Providers Health care CZ and medical staff are absolute Scot-free for even-in the secret continual civic war the Physicians CZ contrary Patients CZ with Mass yearly repeated similar Mass Medical errors in the standard medical orthopadic processing. In this my explanatory Article, I am deciding solitaire scientist-one-man cz only-because the scientific Truth is interesting for US Medicine only, never for CZ Medicine. Namely I am explaining the next Explanatory Orthopadic Case about false Diagnostics and with false choice the Treatments Necrosis of femoral Head both my legs - as an pensioner The State investigator of mortal occupational cases of Ministry for Labour and Social Affairs Czech Republic, so as a needless crippled too and just dying orthopadic Patient „LEGE ARTIS CZ“ too-after to late diagnostics Necroses of Femoral Head with the false choice 3 needless following Treatments of illegal Orthopadic surgeries THA when there were recommended advanced more carefully and safety recommended NON-surgical treatments in time - by the World Encyclopaedic Orthopadic Literature.

I have unofficial studied the contextual sufficient details of Human Orthopady in Necrosis of Femoral Head, Traumatology functional collapsing of human joints, principles of non-surgical treatments of Necroses femoral Head, The recommended classic arthroplastic light Surgeries to repair Human joints, comparing preferences Method Magnetic resonance and limits in RTG imaging in Diagnostics of necrosis in Hip joints, Clinic mandatory Planning of standard individual Implanting Total Hip Arthroplasty, usual patient risks of Full Anaesthesia, Neurologic transplantation and right timing of neurosurgical reconstruction the Nervous Ischiadici by full plegia in Traumatology cases, the typical complications Surgeries by Patient over Age 65+ by THA, Infects dangerous in Surgery Halls, Infect risks in post operational orthopedic implanting, Typical dangerous Hemorrhagic preliminary Infects of Orthopedic Patients, typical preoperational and post operational risks and Health complication by set THA with non-cemented principles the Hip Artificial joints, rational post operational transfusions of Blood, prescriptions of Medicaments with risk of chemical mutual interactions, Posttraumatic Shocks on surgeon Halls, typical fatal mistakes of interpretation in Geometry $3 \mathrm{D}$ in Orthopedic Radiology by THA, Biomechanics and Designing functional sets THA by Producer Tests, Biostatistics analyses THA patient by returning locomotion abilities with modelling optimal possibilities by computer support of modelling, harmonised Laws EU/CZ in fields the Occupational Health and Safety, harmonised European Standards for the parallelly usage of rules the Directive Machinery, Directive 93/42/EEC Medical Devices-Research, Development, finished Testing new product sets THA, Regulation the Usage and State surveillance of usage Orthopadic Implants THA in common Market EU by Providers Health care, functional collapsing Implants, repairing.

Rehabilitation support, physiatry supporting praxes for post operational locomotion abilities of Patient after THA, Statistic Growing Risks of repeated proximal fatal breaking of Femoral bones after repeated THA surgeries by happened fallen. The Duration of safety product lives in sets THA in long being safety patient usage for probable more as 20 years, Carcinomic risks by metallic and micro invasive PVC materials surfaces in frontiers the components of the Artificial joints with surface of cavities of femoral bones, plevis hip sockets, destructions of the functional Technician Quality of mechanical Anchoring of centric placements of components THA in hip bones, Catastrophic preoperational situations "Ad hoc" on the Surgeon Halls by implanting THA. Yes, I am just simulating personally in this Article-the perfect educated, structured and working decision making Team of experts for usage orthopadic sampling in Criminal analyses in the primary Demesne: "The Statistic Unique entity"-with explanation the decision making by my Method S_T_A_R_S, by The Utility model 21532 CZ 2010.

As I unnecessarily lost my left hip in Dg. the femoral head necrosis. At the beginning of 2004, I was 59 years old, I was poorly trained with a new Surface Sleigh sweep in the snow-I dropped from the height of my buttock where there was a big blood swelling about 7 centimetres in diameter. The pains did not lose even after a few months, it had only solid edges. After a few months, my left hip began to collapse, sometimes I was eliminated for a few minutes from locomotion, then it managed to overcome the light massage and another attack of pain inside my hip was repeated until the next day. As an inquisitive patient, I lectured at the Prague Municipal Library to study the Orthopedic Medical Encyclopaedia, where I personally suspected that it might be Necrosis of the left femoral head, according to the typical symptoms of the patient's troubles. 
So, I went to the ambulance of my practical Mrs. Dr. Physician and I asked her to provide me with a request for X-ray examination of the hip at the Orthopedic Clinic of the Faculty Hospital Královské Vinohrady in Prague 10. The X-ray analyst carefully listened to me and looked at the X-ray image from the beginning of September 2004, there are no obvious destructive changes on the X-ray of the hip, but that does not mean that my patient's situation is very serious. She did not ask me to bring a new order for the Distant Magnetic Resonance to detect the initial phase of Femoral Necrosis, but she sent me for further examination at the Neurology Clinic of the same hospital. The Prime Dr. of Neurology, me signed carefully scratched the hip and skeletal functions, but he did not send me again to the desired differential diagnosis of femoral head necrosis using MRI-because he was mistakenly assumed-that it was the cause of spinal nerve ending-with casual both-hip and back pains. Procedure Neurology was not expertly wrong but ignored my initial suspicion about of Necrosis of left femoral head.

The neurologist has decided Treatment-that the painful state should bridle local anaesthesia with a syringe inserted directly at the end of the spine-in the hope that painful inflammation in the spine, as well as the left hips will calm down and the hip joint will functionally revitalize again. The origin premise of neurologist was wrong for always worse Patient Health status in duration of 4 weeks. There was a clear mistake that Neurology kept on his primary hypothesis about Diagnosis, but my patient hypothesis did not at least do the additional differential diagnosis with MRI, my patient council did not take seriously. It is for these reasons that during the tragic 4 weeks the surface sliding surface of the hip joint was destroyed. On the next X-ray image at the beginning of November 2004, the revitalization of the joint function was completely impossible without at least a shorter surgical orthopedic procedure.

According to the Orthopaedics Encyclopaedia, the first stages of the development of necrosis of the femoral head are easily naturally revitalized-only the basic limitations of locomotion with bedtime and the use of revitalizing dietary and medical supplements, with hip autosomes and controlling effects by MRI after 5 weeks. In my private case of orthopedic and already significant damage of the hip joint, the application of Total endoprosthesis is possible only in the highest stage of the $5^{\text {th }}$ stage, but the Orthopedic Clinic of the Faculty Hospital Královské Vinohrady, Prague 10 CZ, offered me the term of Surgeon THA till in May 2005, when I could not walk from the place. That made me looks for a next Orthopedic Promotion at the Regional Regional Hospital Mladá Boleslav where I was implanted with an artificial joint contact $\mathrm{S}$, non-cemented, already on January 4, 2005.

The operation of the hip TEP was performed properly and functionally, but unnecessarily about 5 years earlier before it was for me Patient-friendly - because none of the Orthopedists, Radiologists and Healers-could not use the recommended advanced diagnostic and therapeutic procedures for the diagnosis of femoral head necrosis by Orthopedic Encyclopaedia-nor on the scale and knowledge's of a typical orthopedic educated patient with a right defined probable Dg. According to a sample of about random choice about 50 cases Patient Cluster with the femoral Head with Necrosis in a like hood Orthopadic Clinic... it can be seen with statistic evidence today's trends: - that Orthopadic Clinics, Orthopedists and Radiologists always prefer large surgical orthopadic procedures on which the Orthopedic Clinic and Hospitals can sneeze, including doctors, while the interests and preference of the ORTHOPEDIC PATIENTS are else-AFTER THE PERFECT LIFESTYLE TILL AGES 90+, WITH LOCOMOTIVE AND PERSONAL MENTAL HIGH PRIVATE SAFETY CONDITIONS-always with consistently prudency and eliminating the needless Patient risks, eliminate needless use of local and general anaesthesia by preliminary THA including, in no case there are a independent supervisions of the Health Assurances, or the Providers of health services in orthopedics in hospital nets OECD-BEST RELIABLE DECIDINIG AND SHARING BEST MEDICAL EXPERIENCES IN PRINCIPLES "Based Evidenced Medicine" NOT WARRANT! The Profits of Providers Health care are priorities

How I lost my right hip joint, how could I get crippled and why I'm dying. After about 8 months in August 2005, I began to experience similar problems with necrosis of the femoral head even on the right hip. My Orthoepy MUDr. František Vurm, who has already operated the left hip-He recommended only hip pain pills to limit, which did not solve the essence of the orthopedic principal problem. His fundamental view was - to passively wait until the advanced necrosis of the femur destroys the right hip joint too - and then, finally to use the THA again. He did not think of any more moderate rescue carefully procedures for my hip - I suppose, probably because he did not use any nonsurgical healing processes or mild curative surgical arthroplastic processes. Of course, he did not manage them practically with skills - and perhaps He never read anything about them from the last Czech Orthopadic Encyclopedia since 2005 .

The orthopedic encyclopaedia literally describes full List of orthopadic diagnoses and full variants of treatment methodswhich the unique orthopedic routine does not manage with skills and therefore cannot recommend to its patients for else Orthopedic concurrency in medical Branch. If you want to make a good next profit by next hip Total Hip Arthroplasty. No professional critic from the position of his experienced colleagues-the Orthopedists, does not expect it. Especially in the elderly, it is often with much more advantageous to replicate about number about 200 TEP hip annually from the point of view of the older Orthopaedist to older Patient in the region-rather than trying to risk the easier and cheaper light surgeon repairing of the hip joint for the individual Patient-maybe which he saw similar extraordinary problems twice times about 20 years ago - when he was studying Orthopedics firstly.

All Orthopaedist are keeping mostly trendy-to more frequent the large surgeon standard HIP with Total Arthroplasty, despite there are for Patients frequently growing risks from full Anesthesia, worse rehabilitation, more risks on the Surgeon hall, etc. The fatal legal, technical, medical mistakes of my Orthopaedic MUDr. František Vurm-it was ignoration my preliminary declination the 
femoral neck on the right femoral bone, which was diagnosed by the Radiolog from the Central Medical Radiology Clinic of the Faculty Hospital Královské Vinohrady Praha 10-about 3 weeks before Surgery THA-it means before date November 13, 2007 in the Regional Hospital Mladá Boleslav with explicit results by written too!

By the Directive 93/42/EEC Medical Devices, there are technical requirements including mandatory duties to create "Individual Clinic Processing Plan of Surgery THA", because the individual qualities of the Forms, Positional placement and the individual structure of hip bones-there are limiting Input mandatory information about Patient Health Status including, we should probe the useable fitted placement of the choice components set THA on the preliminary RTG Hip images with mandatory comparison with the firm radiologic etalons of components in Geometry 3D on the Orthopedic screen, with the same scaling...there are mandatory specific planning processing by the Dysplastic Declination of femoral neck....because the separation of dysfunctional forms the natural Head of joint....must be with individual surgeon cat, there must be regards to rasping the specific socket on the proximal femoral cavity...for planning the placement of the stem...Only idiot or Murder...could be able to begin the illegal Surgeon Work THA on the Surgeon Hall "blendy"! Only Group of Murders with graduating as Orthopads MUDrs, could say...it is working Lage artist CZ, including when the MUDr. František Vurm wasn't able to detect the false rasped the proximal femoral socket with false hole - with Illegal destruction integrity of my femoral bone ...despite he should been mandatory controlling by the Product in void with specific Orthopedic instrument full integrity by details in Certification of Users THA Contact S Non-cemented, but he didn't diagnosed tragic hole in the femoral socket.
When MUDr. František Vurm illegal finished the assembling the set THA-Bi contact S, Non-cemented, Dimension $13 \mathrm{~mm}$, Type $\mathrm{N}$ from the B. Braun Germany...out of mandatory accuracy in coaxial ties the femoral bone and there Stem....out of tolerance +/- 1 grads... when he placed the stem illegally crummy ....with declination in the sagittal Direction (the same Plaine and declination of the axes of the Stem on 13,68 grads- Direction as the Imaging RTG since the November 16, 2007). Only Idiots or Murders could say „It is no to detect-illegal declination on the RTG since the date November 16 , 2007“. We should observe the post operational Image not illegally „in the hand and with intuitive views “! We should take always the Firm installing radiological masks and on the Orthopaedic screen, we should comparing the placement on the Image with this radiologic firm etalons.....and when the spice of the used stem in the coordinates Xi, Yi, Zi we were controlling....by the Preliminary Planning.... but there are in the FALSE DEPTH! The spice it is about 5,8 millimetres in femoral axes „virtually shorten axes of the Stem "-Only idiots or my Murders, could say again by Court dealing....it was LEGE ARTIS CZ!

No, there are by the goniometric Algorithm to evidence the same declination 13.68 Grads definitely, as we could see the same declination on the next post operational RTG Image since the date November 28, 2007 before Reoperation THA! It was Crash FAUSSE ROUTE STEM of the false illegal assembling product „CE“, there were no functional mandatory abilities by official testing of implanted product „CE“ on the surgery hall since date November 13,2007 !

Only idiots or my Murders could say again.... "You are probable crippled by the post operational patient happened fallen, all medical orthopaedic workflow were realised LEGE ARTIS CZ", by the meaning medical staff and so as by The Knowing Medical Institute-it is FALSUM and the Complot against the Patient, it was accepted by the Judge!

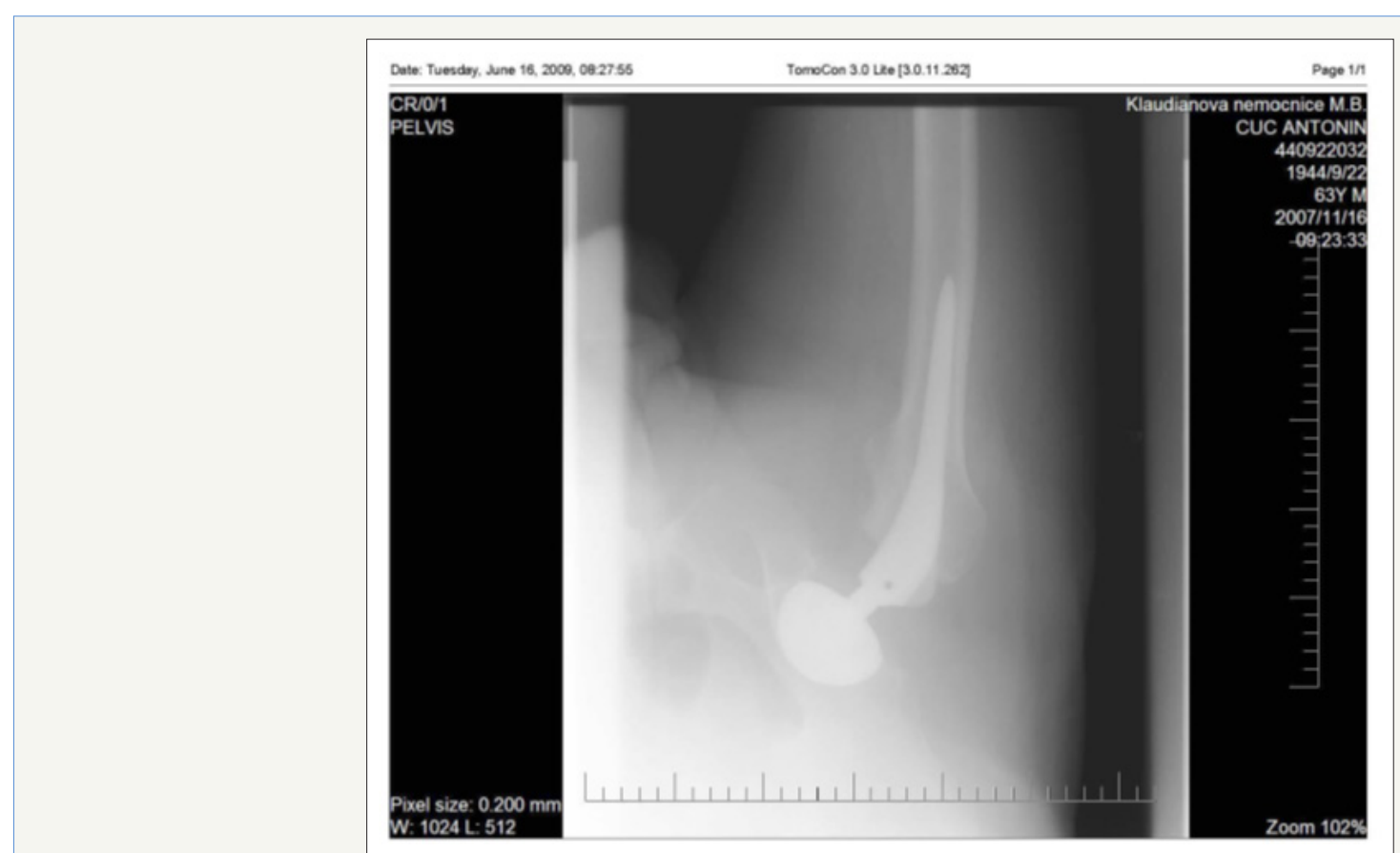

Figure 1 


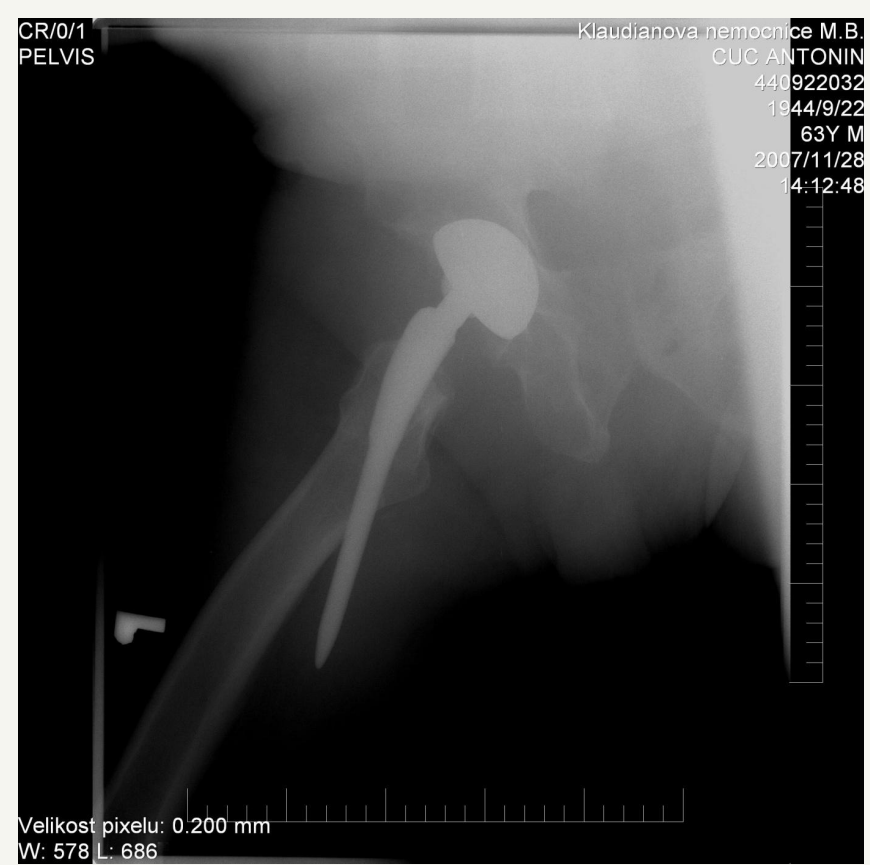

Figure 2

There are absolutely excluded to substitute the real Phenomenon's and Biomechanics, Geometry, Radiologic evidences from Tragic Crash FAUSSE ROUTE in the surgeon Hall since date November 13, 2007-see the next 2 post operational RTG Images“ and the False Medical Hypotheses from the Court Medical Message from the date March 27, 2012 from the Knowing Medical InstituteThe Central Army Hospital Prague CZ Post operational patient Fallen with perfect Lege Artis CZ the Provider Health Care-the Regional Hospital Mladá Boleslaw with medical staff-Orthopaedist MU Dr. František Vurm, prime MUDr. Pavel Šedivý. The False Medical Court Message was signed by the Head of The Central Army Hospital Prague CZ-Prof. MU Dr. Miroslav Zavoral Dr Sc., so as the Head of the Army Forensic Clinic prime MUDr. Miloš Sokol Ph. D., No. 36 C 181/2009-123 since March 27, 2012 (Figure 1 \& 2).

The mortal orthopadic injuring from the Regional Hospital Mladá Boleslav was completed by in forced extremely repeated RTG ionisation in January 2012, when the summary radiation caused me the Leukaemia B-CLL, perfect detect on the Blood parameters since October 2012 and as the mortal diagnose it was diagnosed perfect with details in Haematology of Institute of Clinic and Experimental Medicine Prague 4, CZ from the February 2013.

In just time my orthopaedic diseases are continuing with drastic limiting my locomotion abilities, with full pelagic destruction of the nervous Ischiatic in my right under knee since the November 13, 2007 for ever, with in forced next reoperation THA in November 30,2007 , with many repeated annually risky patients fallen with $8 x$ more probable mortal repeated the breaking proximal femoral bone as I tis usual in Cluster Patient with standard THA. With leukemic destructions with redundant in forced extremal ionisationout of Court Judicial Question "IT WAS MEDICAL IMPLANTING PROCESSING LEGE ARTIS CZ?"
All my Murders are smiling me in Complot among the Culprits and Knowing Court Experts "MUDrs "with the worldwide famous evaluation Health care against Patients CZ, it is calling „LEGE ARTIS CZ“! There are after false illegal usage Medical Devices about 40 thousand Patients CZ needless heavy injured and mostly preliminary casual dying Antaly.

None of State Penalty Offices CZ could renewed the Penalty Court Trial against my Murders with perfect respects medical official Tittles MUDr CZ, I have asked it since the July 2013 vainly! The Judge JU Dr. Vojtěch Cepl from the Regional Court Prague was perfect informed before Court dealing by my Criminal evidences by written in my recommended Letter since the July 17, 2012, despite the accepting of False Medical Court Message was realised officially in September 17, 2012, the false criminal argumenta Judgement was derived from the false Court Medical Message as No. 36 C 181/2009-221 since September 24, 2012 -„Patient post operational Unhappy, no Fuse route stem“. I was asking about Civic Legal Support for my Human Rights CZ to justified Court processing with the justified and verified Criminal evidences only by Penalty Court Trial, including asking the Ombudsman CZ about support, but It is without a like hood official Answering till todays. I have no money and no Healthy to defend my Human Rights in the European Court for Human Rights EU/CZ in the Strasbourg, but I hope in your empathy and rational reuse to decide the Method S_T_A_R_SStatistical Retrieval and search of sufficient information to Mass usage for repeated similar strategic decision making with risk and computer support, 21532 CZ 2010.

\section{References}

1. McNally EG, Rees JL (2007) Imaging in shoulder disorders. Skeletal Radiol 36(11): 1013-1016. 
2. Jacobson JA (2011) Shoulder US: Anatomy, technique, and scanning pitfalls. Radiology 260(1): 6-16.

3. Beggs I (2011) Shoulder ultrasound. Semin Ultrasound CT MR 32(2): 101-113.
4. Smith TO, Back T, Toms AP, Hing CB (2011) Diagnostic accuracy of ultrasound for rotator cuff tears in adults: A systematic review and meta-analysis. Clin Radiol 66(11): 1036-1048.

5. Abdullaev RY, Dzyak GV, Dudnik TA (2010) Ultrasonography of the shoulder joint. Kharkov, Ukraine. (c) (i) $\begin{aligned} & \text { Creative Commons Attribution } 4.0 \\ & \text { BY }\end{aligned}$

For possible submissions Click Here
Submit Article

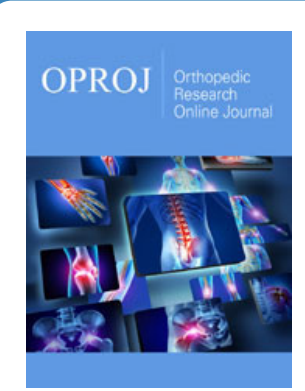

\section{Orthopedic Research Online Journal}

Benefits of Publishing with us

- High-level peer review and editorial services

- Freely accessible online immediately upon publication

- Authors retain the copyright to their work

- Licensing it under a Creative Commons license

- Visibility through different online platforms 